УДК 004.052, 519.179.2

\title{
A Verification Method for a Family of Multi-agent Systems of Ambiguity Resolution»
}

\author{
Natalia Garanina (A.P. Ershov Institute of Informatics Systems), \\ Elena Sidorova (A.P. Ershov Institute of Informatics Systems)
}

In the paper we describe a verification method for families of distributed systems generated by context-sensitive network grammar of a special kind. The method is based on model checking technique and abstraction. A representative model depends on a specification grammar for family of systems. This model simulates a behavior of the systems in such a way that properties which hold for the representative model are satisfied for all these systems. We show using this method for verification of some properties of multiagent system for resolution of context-dependent ambiguities in ontology population.

Keywords: model checking, context-sensitive network grammar, multi-agent systems, abstraction

\section{Introduction}

The motivation of our work is the ambiguity resolution problem in the frame of ontology population from natural language texts. In [6] we describe text analysis algorithms producing a system of information agents. But features of natural language cause ontology population ambiguities, which these agents have to resolve. We proposed to evaluate the cardinality of agents' contexts, i.e. how much an agent is related with the other agents of the resulting system via the information contained in it, and to mark the agents the most integrated in the text. We developed an ambiguity resolution algorithm [5], removing the less integrated agents from the system.

All agents in parallel perform rather complicate protocols with periodic local synchronizations. Hence, it is reasonable to use formal verification methods for proving correctness of the algorithm. We choose model checking technique for a particular multi-agent system. We verify rather specific multi-agent system of conflict resolution. The works on multi-agent systems usually focus on the behavior of agents, methods of communication between agents, knowledge and belief of an agent about environment and other agents, etc [4,9]. Works about conflict resolution process usually consider the process in terms of the behavior of the agent depending 
on its internal state, reasoning and argumentation methods etc. [8]. The dynamics of the agents connections is not a subject of these researches. There are papers related to the dynamics of weighted connections, but they are not the typed and their changes does not affect the internals of the agent [7]. On the other hand there are works on the study of social networks, in which the agents are connected by the typed connections, but their weight does not matter [1]. To the best of our knowledge, there are no works on model checking for a conflict resolution algorithm of the suggested type.

Model checking technique is widely used for verification of distributed and multiagent systems [2]. In our case we would like to verify not a particular agent network, but infinite family of such systems. For verification of infinite network families the model checking method was suggested in [3]. This method is based on using a context-free network grammar generating families of distributed systems, and on abstraction by finite automata. The idea of the method is to construct an invariant network based on a given grammar. This invariant simulates behavior of all systems in the family and is consistent with abstract functions associated with properties to be verified which are expressed by branching time logic $\forall C T L$. Due to consistent simulation, properties holding for the representative invariant also holds for all systems in the family. But authors studied context-free grammars only, while our model of the multiagent system is generated by a context-sensitive grammar of a special kind. In the paper we define such network grammar by adding notions of a quasi-terminal and a merging operator to the standard definition. We show that this verification method still can be used for network families generated by the new grammar.

The rest of the paper is organized as follows. The next section 2 gives base definitions. Section 3 presents results on a new merging operator, used in our context-sensitive network grammar. Section 4 describes using our method for the multiagent system of ambiguity resolution. We conclude in the last section 5 with a discussion of further research.

Acknowledgments. The research has been supported by Russian Foundation for Basic Research (grant 15-07-04144) and Siberian Branch of Russian Academy of Science (Integration Grant n.15/10 "Mathematical and Methodological Aspects of Intellectual Information Systems").

\section{Base Definitions}


Let us give necessary definitions from [3] in a modified form. Modification concerns a merging operator and quasi-terminals in a network grammar.

\section{Definition 1.}

A Labeled Transition System (LTS) is a structure $M=\left(S, R, A C T, S_{0}\right)$, where

- $S$ is the set of states,

- $S_{0} \subseteq S$ is the set of initial states,

- $A C T$ is the set of actions, and

- $R \subseteq S \times A C T \times S$ is the total transition relation, such that for every $s \in S$ there is action $a$ and state $s^{\prime}$ for which $\left(s, a, s^{\prime}\right) \in R\left(\right.$ denote as $\left.s \stackrel{a}{\longrightarrow} s^{\prime}\right)$.

Let $L_{A C T}$ be the class of LTSs whose set of actions is a subset of $A C T$. Let $L_{(S, A C T)}$ be the class of LTSs whose state set is a subset of $S$ and whose action set is the subset of $A C T$. Let $A C T_{1}, A C T_{2} \subseteq A C T$. Let we are given two LTSs $M_{1}=\left(S_{1}, R_{1}, A C T_{1}, S_{0}^{1}\right)$ and $M_{2}=\left(S_{2}, R_{2}, A C T_{2}, S_{0}^{2}\right)$ in the class $L_{A C T}$.

\section{Definition 2.}

A function $\|: L_{A C T} \times L_{A C T} \mapsto L_{A C T}$ is called a composition function iff $M_{1} \| M_{2}$ has the form $\left(S_{1} \times S_{2}, R^{\prime}, A C T_{1} \cup A C T_{2}, S_{0}^{1} \times S_{0}^{2}\right)$.

A function $\cup: L_{A C T} \times L_{A C T} \mapsto L_{A C T}$ is called a merging function iff $M_{1} \cup M_{2}$ has the form $\left(S_{1} \cup S_{2}, R^{\prime}, A C T_{1} \cup A C T_{2}, S_{0}^{1} \cup S_{0}^{2}\right)$.

The definition of $R^{\prime}$ depends upon the exact semantics of the composition and merging function. Let $S^{i}$ be words of length $i$ with $S$ as the alphabet.

\section{Definition 3.}

Given a state set $S$ and a set of actions $A C T$, any subset of $\bigcup_{i=1}^{\infty} L_{\left(S^{i}, A C T\right)}$ is called a network on the tuple $(S, A C T)$.

We give a definition of a context-sensitive network grammar with quasi-terminals (CSNQgrammar) to describe networks, which is the modified definition of a context-free network grammar from [3]. The set of all LTSs derived by a network grammar forms a network which is an LTS also. Let $S$ be a state set and $A C T$ be a set of actions. CSNQ-grammar $G=$ $(T, Q t, t, N, P, S)$ is a grammar, where

- $T$ is a set of terminals, each of which is an LTS in $L_{(S, A C T)}$, these LTSs are sometimes referred to as basic processes, 
- $Q t$ is a set of quasi-terminals, each of which is an LTS in $L_{(S, A C T)}$, their merging gives an LTS,

- a mapping $t: Q t \mapsto T$ associates quasi-terminals to terminals,

- $N$ is a set of nonterminals, each nonterminal defines a network,

- $P$ is a set of production rules of the following forms:

$-A \longrightarrow B \|_{i} C$, where $A \in N$, and $B, C \in T \cup Q t \cup N$, and $\|_{i}$ is a composition function.

$-\left\{A_{1}, \ldots A_{n}\right\} \longrightarrow t\left(A_{1}\right) \cup_{i} \ldots \cup_{i} t\left(A_{n}\right)$, where $A_{j} \in Q t$, and $\cup_{i}$ is a merging function.

- $S \in N$ represents the network generated by the grammar.

Note, that this grammar is context-free with respect to composition functions and contextsensitive with respect to merging functions.

In order to express properties of a model composed from finite, but unspecified number of LTSs, we define a finite automaton on alphabet $S$.

\section{Definition 4.}

$D=\left(Q, q_{0}, \delta, F\right)$ is a deterministic automaton over $S$, where

- $Q$ is the set of automaton states,

- $q_{0} \in Q$ is the initial state,

- $\delta \subseteq Q \times S \times Q$ is the transition relation,

- $F \subseteq Q$ is the set of accepting states, and

- $L(D) \subseteq S^{*}$ is the set of words accepted by $D$.

We use finite automata over $S$ for specification of atomic state properties. Let $D$ be an automaton over $S$. State $s$ satisfies $D(s \models D)$ iff $s \in L(D)$. A specification language is a universal branching temporal logic $\forall C T L$ [3] with finite automata over $S$ as the atomic formulas. Syntax of $\forall C T L$ consists of formulas that are composed of Boolean constants, atomic formulas, connectives $\neg, \vee, \wedge$, and branching time modalities $\mathbf{A X} \varphi, \mathbf{A G} \varphi$, and $\varphi \mathbf{A U} \psi$ with standard semantics.

Recall definitions for abstract LTS from [3]. For the simplicity, here the specification language contains a single atomic formula $D$. Given an automaton $D=\left(Q, q_{0}, \delta, F\right)$ and a word $w \in S^{*}$ the function induced by $w$ on $Q, f_{w}: Q \mapsto Q$, is defined by $f_{w}(q)=q^{\prime}$ iff $q \stackrel{w}{\longrightarrow} q^{\prime}$. Note that $w \in L(D)$ if and only if $f_{w}\left(q_{0}\right) \in F$. Two states $s$ and $s^{\prime}$ are equivalent $s \equiv s^{\prime}$ iff $f_{s}=f_{s}^{\prime}$. The function $f_{s}$ is called the abstraction of $s$ and is denoted by $h(s)$. Relation $\models$ is extended to abstract states: $h(s) \models D$ iff $f_{s}\left(q_{0}\right) \in F$. Hence $s \models D$ iff $h(s) \models D$. 
Let $F_{D}$ be the set of functions corresponding to the deterministic automaton $D$. The abstraction function $h$ extended to $F_{D}$ is defined by $h(f)=f$ for $f \in F_{D}$ and extension the function $h$ to $\left(S \cup F_{D}\right)$ is $h\left(\left(a_{1}, a_{2}, \ldots, a_{n}\right)\right)=h\left(a_{1}\right) \circ \ldots \circ h\left(a_{n}\right)$. From now on we consider LTSs in the network $N$ on the tuple $\left(S \cup F_{D}, A C T\right)$.

Definition 5. (of abstract LTS)

Given an LTS $M=\left(S^{i}, R, A C T, S_{0}\right)$ in the network $N$, the corresponding abstract LTS is defined by $h(M)=\left(S^{h}, R^{h}, A C T, S_{0}^{h}\right)$, where

- $S^{h}=\left\{h(s) \mid s \in S^{i}\right\}$ is the set of abstract states,

- $S_{0}^{h}=\left\{h(s) \mid s \in S_{0}\right\}$, and

- the relation $R^{h}$ is defined as follows. For any $h_{1}, h_{2} \in S^{h}$, and $a \in A C T$ : $\left(h_{1}, a, h_{2}\right) \in R^{h} \Leftrightarrow \exists s_{1}, s_{2}\left[h_{1}=h\left(s_{1}\right) \wedge h_{2}=h\left(s_{2}\right) \wedge\left(s_{1}, a, s_{2}\right) \in R\right]$.

$M^{\prime}$ simulates $M$ (denoted $\left.M \preceq M^{\prime}\right)$ iff there is a simulation preorder $E \subseteq S \times S^{\prime}\left(\left(s, s^{\prime}\right) \in E\right.$ denoted $s \preceq s^{\prime}$ ) that satisfies the following conditions: for every $s_{0} \in S_{0}$ there is $s_{0}^{\prime} \in S_{0}^{\prime}$ such that $s_{0} \preceq s_{0}^{\prime}$. For every $s, s^{\prime}$, if $s \preceq s^{\prime}$ then

- $h(s)=h\left(s^{\prime}\right)$, and

- for every $s_{1}$ such that $s \stackrel{a}{\longrightarrow} s_{1}$ there is $s_{1}^{\prime}$ such that $s^{\prime} \stackrel{a}{\longrightarrow} s_{1}^{\prime}$ and $s_{1} \preceq s_{1}^{\prime}$.

\section{The Merging Operator in the Verification Framework}

The first two propositions of the following lemma were proved in [3], the last is proved below:

\section{Lemma 1.}

1. $M \preceq h(M)$, i.e., $h(M)$ simulates $M$.

2. If $M \preceq M^{\prime}$, then $h(M) \preceq h\left(M^{\prime}\right)$.

3. $M \cup M^{\prime} \preceq h(M) \cup h\left(M^{\prime}\right)$

Proof of (3) is obvious: $M \cup M^{\prime} \preceq h\left(M \cup M^{\prime}\right)$ due to (1), and $h\left(M \cup M^{\prime}\right)=h(M) \cup h\left(M^{\prime}\right)$.

The following theorem about satisfiability of properties in an LTS and its simulator was proved in [3] and holds for our new framework.

\section{Theorem 1.}

Let $\varphi$ be a formula in $\forall C T L$ over the atomic formula $D$. Let $M$ and $M^{\prime}$ be two LTSs such that $M \preceq M^{\prime}$. Let $s \preceq s^{\prime}$. Then $s^{\prime} \models \varphi$ implies $s \models \varphi$. 


\section{Definition 6.}

A merging or composition operator $\bullet \in\{\cup, \|\}$ is called monotonic with respect to a simulation preorder $\preceq$ if and only if given LTSs such that $M_{1} \preceq M_{2}$ and $M_{1}^{\prime} \preceq M_{2}^{\prime}$, we have that $M_{1} \bullet M_{1}^{\prime} \preceq$ $M_{2} \bullet M_{2}^{\prime}$. A network grammar $G$ is called monotonic if and only if all rules in the grammar use only monotonic composition and merging operators.

We modify a synchronous framework from [3] with results for the merging operator. Let models be a form of LTSs, Moore machines $M=\left(S, R, I, O, S_{0}\right)$ such that inputs $I$ and outputs $O$ must be disjoint. In addition, they have a special internal action denoted by $\tau$. The set of actions is $A C T=\{\tau\} \cup 2^{I \cup O}$, where each noninternal action is a set of inputs and outputs. A transition $s \stackrel{a}{\longrightarrow} t$ from $s$ in a machine $M$ with $a=i \cup o$ such that $i \subseteq I$ and $o \subseteq O$ occurs only if the environment supplies inputs $i$ and the machine $M$ produces the outputs $O$.

Naturally, for the merging operator inputs and outputs of merging machines must be disjoint also. Let $I \cap O^{\prime}=\emptyset$ and $O \cap I^{\prime}=\emptyset$. The merging of $M$ and $M^{\prime}, M^{\prime \prime}=M \cup M^{\prime}$ is defined by

- $S^{\prime \prime}=S \cup S^{\prime}$,

- $S_{0}^{\prime \prime}=S_{0} \cup S_{0}^{\prime}$,

- $I^{\prime \prime}=I \cup I^{\prime}$ and $O^{\prime \prime}=O \cup O^{\prime}$, and

- $s^{\prime \prime} \stackrel{a^{\prime \prime}}{\longrightarrow} s_{1}^{\prime \prime}$ is a transition in $R^{\prime \prime}$ iff the following holds: $s^{\prime \prime} \stackrel{a}{\longrightarrow} s_{1}^{\prime \prime}$ is a transition in $R$ and $s^{\prime \prime} \stackrel{a^{\prime}}{\longrightarrow} s_{1}^{\prime \prime}$ is a transition in $R^{\prime}$ for some $a, a^{\prime}$ such that $a^{\prime \prime}=a$ or $a^{\prime \prime}=a^{\prime}$.

\section{Lemma 2.}

The merging $\cup$ is monotonic with respect to $\preceq$.

Proof. Let $M=\left(S, R, I, O, S_{0}\right), M_{1}=\left(S_{1}, R_{1}, I_{1}, O_{1}, S_{1,0}\right), M^{\prime}=\left(S^{\prime}, R^{\prime}, I^{\prime}, O^{\prime}, S_{0}^{\prime}\right), M_{1}^{\prime}=$ $\left(S_{1}^{\prime}, R_{1}^{\prime}, I_{1}^{\prime}, O_{1}^{\prime}, S_{1,0}^{\prime}\right)$ be four Moore machines. Assume that $M \preceq M_{1}$ and $M^{\prime} \preceq M_{1}^{\prime}$. Let $E \subseteq S \times S_{1}$ and $E^{\prime} \subseteq S^{\prime} \times S_{1}^{\prime}$ be the corresponding simulation relations. We prove that $M \cup M^{\prime} \preceq M_{1} \cup M_{1}^{\prime}$.

We say that $\left(s^{\prime \prime}, s_{1}^{\prime \prime}\right) \in E^{\prime \prime}$ iff $\left(s^{\prime \prime}, s_{1}^{\prime \prime}\right) \in E$ or $\left(s^{\prime \prime}, s_{1}^{\prime \prime}\right) \in E^{\prime}$. We show that $E^{\prime \prime}$ has the required properties. It is clear from the definition that given state $s_{0} \in S_{0} \cup S_{0}^{\prime}$, there exists $s_{0,1} \in S_{0,1} \cup S_{1,0}^{\prime}$ such that $\left(s_{0}, s_{0,1}\right) \in E \cup E^{\prime}$.

Assume that $\left(s, s_{1}\right) \in E \cup E^{\prime}$.

(1) By assumption, we have that $h(s)=h\left(s_{1}\right)$.

(2) Let $s \stackrel{a^{\prime \prime}}{\longrightarrow} t$ be a transition in $M \cup M^{\prime}$. This means that there exists transition $s \stackrel{a}{\longrightarrow} t$ in $M$ or transition $s \stackrel{a^{\prime}}{\longrightarrow} t$ in $M^{\prime}$ such that $a^{\prime \prime}=a$ or $a^{\prime \prime}=a^{\prime}$. By definition there exists $t_{1} \in S_{1} \cup S_{1}^{\prime}$ 
such that $s_{1} \stackrel{a}{\longrightarrow} t_{1}$ or $s_{1} \stackrel{a^{\prime}}{\longrightarrow} t_{1}$, where $\left(t, t_{1}\right) \in E$ or $\left(t, t_{1}\right) \in E^{\prime}$. Therefore, $s_{1} \stackrel{a^{\prime \prime}}{\longrightarrow} t_{1}$ and $\left(t, t_{1}\right) \in E^{\prime \prime}$. The proof is thus complete.

The notion of a representative give us a way to construct a simulation invariant. Given a CSNQ-grammar $G$, we associate with each symbol $A$ of the grammar a representative process $\operatorname{rep}(A)$. Let us adopt the definition of a monotonicity property for a set of representative processes of CSNQ-grammar:

- for every terminal and quasi-terminal $A$ : $h(\operatorname{rep}(A)) \succeq h(A)$, and

- for every rule $A \longrightarrow B \| C: h(\operatorname{rep}(A)) \succeq h(h(\operatorname{rep}(B)) \| h(\operatorname{rep}(C)))$.

We extend the proof of the following theorem on context-free network grammar from [3] to CSNQ-grammars:

\section{Theorem 2.}

Let $G$ be a monotonic grammar and suppose we can find representatives for the symbols of $G$ that satisfy the monotonicity property. Let $A$ be a symbol of the grammar $G$, and let $a$ be an LTS derived from $A$ using the rules of the grammar $G$. Then, $h(\operatorname{rep}(A)) \succeq a$.

Proof. We prove that $h(\operatorname{rep}(A)) \succeq h(a)$. Since $h(a) \succeq a$, the result follows by transitivity. Let $A \Rightarrow^{k} a$, i.e., $A$ derives $a$ in $k$ steps. Induction on $k$.

$(k=0)$ Proved in [3].

$(k=1)$ In the case $A, B$ are quasi-terminals in a rule $A, B \longrightarrow t(A) \cup t(B)$ and $a=t(A) \cup t(B)$.

The result follows from the monotonicity property and Lemma 1.

$(k \geq 1)$ Proved in [3].

Verification method is exactly the same as in [3]. Assume that we are given monotonic grammar $G$ and $\forall C T L$ formula $\varphi$ with atomic formulas $D_{1}, \ldots, D_{k}$. To check that every LTS derived by the grammar $G$ satisfies $\varphi$ we perform the following steps:

1. For every symbol $A$ in $G$ choose representative process $r e p(A)$ and construct the abstract LTS $h(r e p(A))$ with respect to the formulas $D_{1}, \ldots, D_{k}$.

2. Check that the set of representatives satisfies the monotonicity property. Theorem 2 implies that for every a derived by the grammar $G, h(\operatorname{rep}(S)) \succeq a$.

3. Perform model checking on $h(\operatorname{rep}(S))$ with specification $\varphi$. By Theorem 1 , if $h(\operatorname{rep}(S)) \models$ $\varphi$, then for all LTSs $M$ derived by the grammar $G, M \models \varphi$.

For finding monotonic representatives we could use an algorithm from [3] setting $\{t(A)\}$ as an initial representative association set of every quasi-terminal $A$. 


\section{Verification of Multiagent Ambiguity Resolution}

A detailed description of the multiagent algorithm for ambiguity resolution in ontology population is given in [5]. In this paper we sketch a communication structure without considering agents' actions on message processing.

Let a set of information agents be given. Some of agents are in a conflict corresponding to some ambiguity. An agent-master constructs a conflict-free set of information agents taking into account integration of conflict agents in the system. This integration is evaluated by computing weights and conflict weights of the agents. A conflict is resolved by removing a weak agent from the system. The agent-master performs the main protocol of constructing the conflict-free set, while the information agents perform protocols of computing their weights.

Every information agent is connected with the master by two-way channel. Information agents are linked with others by labeled connections of two types corresponding their conflict reaction: removing (rem-type) and updating (upd-type). Every labeled connection is acyclic. Processing of every conflict reaction induced by specified connection is considered to be certain base process. An information agent can be union of such processes. This fact specifies a form of a grammar generating family of our multiagent systems for various number of agents connected in various ways. Agents are connected by two-way channels corresponding to these labeled connections. This structure of multiagent network is generated by the following contextsensitive grammar with quasi-terminals $G=(T, Q t, t, N, P, S)$. Let a set of connections be $C=\left\{c_{1}, \ldots, c_{n}\right\}$ and $c_{i}^{k}$ be a connection having conflict type $k \in\{$ rem, del $\}$.

- terminals $T=\{$ master $\} \cup \bigcup_{i=1}^{n}\left\{\right.$ root $_{i}$, inter $_{i}$, lea $\left._{i}\right\} \cup$ vrtxs $^{i}$, and vrtx $^{i}=\{$ vrtx $\mid$ vrtx $=$ inter $_{j}$ or $\left.v r t x=l e a f_{j}, j \in[1 . . n]\right\}$ and $\left|v r t x s^{i}\right|=i$,

- quasi-terminals $Q t=\bigcup_{i=1}^{n}\left\{I N T E R_{i}, L E A F_{i}\right\}$,

- associate mapping $t: Q t \mapsto T$ is defined by $t\left(I N T E R_{i}\right)=$ inter $_{i}$, and $t\left(L E A F_{i}\right)=l e a f_{i}$ for every $i \in[1 . . n]$,

- nonterminals $N=\{S\} \bigcup_{i=1}^{n}\left\{R O O T_{i}, S U B_{i}\right\}$;

- set of production rules $P$ for every $i \in[1 . . n]$ :

1. $S \longrightarrow$ master $\left\|_{m} \operatorname{ROOT}_{1}\right\|_{m} \ldots \|_{m} \operatorname{ROOT}_{n}$

2. $\operatorname{ROOT}_{i} \longrightarrow\left(\operatorname{ROOT}_{i} \|_{c_{i}^{k}} S U B_{i}\right) \bigvee\left(\operatorname{root}_{i} \|_{c_{i}^{k}} S U B_{i}\right)$

3. $S U B_{i} \longrightarrow\left(S U B_{i} \|_{c_{i}^{k}} S U B_{i}\right) \bigvee\left(I N T E R_{i} \|_{c_{i}^{k}} S U B_{i}\right) \bigvee$

$$
\begin{aligned}
& \left(S U B_{i} \|_{c_{i}^{k}} L E A F_{i}\right) \bigvee\left(I N T E R_{i} \|_{c_{i}^{k}} L E A F_{i}\right) \bigvee \\
& \left(\text { inter }_{i} \|_{c_{i}^{k}} S U B_{i}\right) \bigvee\left(S U B_{i} \|_{c_{i}^{k}} \text { lea } f_{i}\right) \bigvee
\end{aligned}
$$




$$
\left(\text { inter }_{i} \|_{c_{i}^{k}} L E A F_{i}\right) \bigvee\left(I N T E R_{i} \|_{c_{i}^{k}} \text { leaf } f_{i}\right) \bigvee\left(\text { inter }_{i} \|_{c_{i}^{k}} \text { lea } f_{i}\right)
$$

4. $\left\{V_{1}, \ldots, V_{m}\right\} \longrightarrow t\left(V_{1}\right) \cup \ldots \cup t\left(V_{m}\right)=v_{r t x}{ }^{m}$, where for every $j \in[1 . . m] V_{j} \in$ $\left\{I N T E R_{i}, L E A F_{i}\right\}$, and if $V_{j}=I N T E R_{i}$ then for every $l \in[1 . . m]$ holds $V_{l} \neq L E A F_{i}$ $(i \in[1 . . n])$.

Parallel composition of agent-processes is synchronous. Protocols for computing weights and conflict weights are highly parallel. Hence it is very important to prove that they terminate and are synchronized properly. Satisfiability of these properties is necessary for correctness of weight computing. Launch of these computing could be modeled by sending tokens.

Every base process is defined by the following state variables:

- Name : int is a name of the process;

- Channel: set of $\{$ name : int; c_type : bool; dir : bool; agn : int; rmvd: bool\}, where name is a label of a connection, c_type is its type, dir is a direction: a child $(\operatorname{dir}=0)$ or a parent $(\operatorname{dir}=1)$ named $a g n$, and $r m v d$ is an absence status;

- Rmvd : bool is an absence status;

- Active : bool is an activity status;

- WasActive : bool is a previous activity status.

In synchronous composition of base processes with different names the corresponding channels of the same name must connect. In merging of processes with the same Name sets of channels and sets of Channel join. Processes with different names cannot be merged and processes with the same Name cannot be composed in parallel. Values of above variables define states of a base process. Its input and output channels correspond to names, types and directions of Channel. Transitions are defined by sending and receiving tokens through the channels. The initial state is (Channel, 0,0,0), where Channel is a nonempty set of channels with Channel.rmvd $=0$, and a number of channels with dir $=1$ does not exceed 1 and a number of channels with $\operatorname{dir}=0$ can be equal to 0.

We would like to verify the following properties expressed by $\forall C T L$. For the protocol of parallel weight computing: $\mathbf{A F}\left(\{\text { wasActive }\}^{*} \wedge \mathbf{A X A F}\{\neg \text { Active }\}^{*}\right)$ (every agent was active, and then all computation will be terminated). For the protocol of conflict weight computing: $\mathbf{A F}\{\neg \text { Active }\}^{*}$ (all computation will be terminated); AG $\{\text { Not2Rmvd }\}^{*}$ (Channels and agents cannot be removed twice). For every atomic formula we construct a finite deterministic automaton. They are a base for abstract functions for states of our systems. Then we should construct a set of consistent representatives for symbols of our grammar. This technique is not 
present here.

\section{Conclusion}

In the paper we present the verification method for families of distributed systems specified by a context-sensitive grammar with quasi-terminals. This method can be used for verification of the multi-agent system of ambiguity resolution in ontology population. Properties of the system are expressed by $\forall C T L$-formulas.

In the near future we plan to implement the suggested method using model checking tool SPIN and give formal proofs of correctness of the ambiguity resolution algorithm. But some properties concerning agent interaction cannot be expressed easily in this framework. This fact is a reason for trying other more expressive formalisms for properties. Other research direction is to extend the method for other types of context-sensitive grammars.

\section{Список литературы}

1. Bergenti F., Franchi E., Poggi A. Selected models for agent-based simulation of social networks // In: Procs. 3rd Symposium on Social Networks and Multiagent Systems (SNAMAS 2011) 2011, pp. $27-32$.

2. Clarke E.M., Grumberg O., Peled D. Model Checking. MIT Press, 1999.

3. Clarke E.M., Grumberg O., Jha S. Verifying Parameterized Networks // In: ACM Transactions on Programming Languages and Systems, Vol. 19, No. 5, September 1997. Pages 726-750.

4. Fagin R., Halpern J.Y., Moses Y., Vardi M.Y. Reasoning about Knowledge. MIT Press, 1995.

5. Garanina N., Sidorova E. An Approach to Ambiguity Resolution for Ontology Population // Proc. of the 24th International Workshop on CS\&P. Rzeszow, Poland, Sep. 28-30, 2015. - University of Rzeszow, 2015, Vol. 1, pp 134-145.

6. Garanina N. O., Sidorova E. A. Ontology Population as Algebraic Information System Processing Based on Multi-agent Natural Language Text Analysis Algorithms//Programming and Computer Software, 2015, V. 41, n.3, pp. 140-148.

7. De Gennaro M.C., Jadbabaie, A. Decentralized Control of Connectivity for Multi-Agent Systems // In: Proc. of 45th IEEE Conference on Decision and Control, pp. 3628 - 3633.

8. Huhns M. N., Stephens L. M. Multiagent Systems and Societies of Agents // In: Multiagent Systems, MIT Press, 1999 pp. 79-120.

9. Wooldridge, M. An Introduction to Multiagent Systems. Willey\&Sons Ltd, 2002. 